\title{
TESTE DA FUNÇÃO TIROIDIANA NA HEPATITE CRÔNICA VIRAL
}

\author{
Mônica NOVIS ${ }^{1}$, Mario VAISMAN ${ }^{2}$ e Henrique Sérgio Moraes COELHO ${ }^{1}$
}

\begin{abstract}
RESUMO - Racional - Foram estudados 125 pacientes com hepatite crônica ativa e cirrose pós-hepatite por virus $B$ ou C, e diferentes graus de acometimento funcional hepático. Objetivo - Traçar um perfil hormonal tiroidiano nesses pacientes. Material e Métodos - Os pacientes foram divididos em quatro grupos: a) 31 com hepatite crônica ativa; b) 41 com cirrose pós-hepatite Child A; c) 35 com cirrose pós-hepatite Child B e d) 18 com cirrose pós-hepatite Child C. Além das dosagens séricas de albumina e bilirrubina, determinação do tempo de atividade da protrombina e avaliação da presença de ascite e encefalopatia hepática, todos foram submetidos a dosagens séricas de triiodotironina (T3), tiroxina (T4), hormônio estimulador da tiróide (TSH), tiroxina livre (FT4), triiodotironina reversa (rT3), cálculo do índice rT3/T3 (IrT3) e realização do teste do TRH. Resultados - Quando se compararam as médias dos resultados dos testes entre os diferentes grupos, observou-se que o T3 foi o exame mais expressivo, gradativamente mais baixo, quanto mais avançada a doença (hepatite crônica ativa: 149,2 $\pm 42,3 \mathrm{ng} / \mathrm{dL}$; cirrose pós-hepatite Child A: 137,4 $\pm 37,2 \mathrm{ng} / \mathrm{dL}$; cirrose pós-hepatite Child B: $88,0 \pm 28,4 \mathrm{ng} / \mathrm{dL}$ e cirrose póshepatite Child C: 41,8 $\pm 21,9 \mathrm{ng} / \mathrm{dl}$ ). Os niveis do TSH, T4 e FT4 foram normais na maioria dos pacientes, porém niveis séricos baixos do T4 (4,5 $\pm 2,0 \mu \mathrm{g} / \mathrm{dL})$ e FT4 (0,7 $\pm 0,4 \mathrm{ng} / \mathrm{dL})$ e elevados do TSH $(7,2 \pm 11,5 \mu \mathrm{IU} / \mathrm{mL}), r T 3(60,8 \pm 52,1 \mathrm{ng} /$ $d L)$ e IrT3 $(2,2 \pm 2,6)$ eram mais freqüentes nos pacientes com cirrose pós-hepatite Child C. O teste do TRH foi normal na maioria dos pacientes. Conclusão - O estudo mostra relação direta entre os níveis séricos baixos do T3 e elevados do rT3 e do IrT3 com o grau de disfunção hepática, segundo a classificação de Child-Pugh.
\end{abstract}

DESCRITORES - Testes de função tiróidea. Hepatite crônica. Cirrose hepática.

\section{INTRODUÇÃO}

Na década de 60 surgiram vários estudos que demonstraram alterações nas dosagens séricas dos hormônios tiroidianos em patologias não-tiroidianas ${ }^{(14,28)}$. Desde então, outros trabalhos foram publicados descrevendo doenças sistêmicas agudas ou crônicas, acompanhadas ou não por desnutrição e caquexia, nas quais o perfeito entendimento da função tiroidiana não era simples, pois havia alterações dos níveis hormonais no sangue, independentemente da presença de patologia endócrina ${ }^{(11,12,18,37)}$.
Anormalidades dos testes de função tiroidiana em associação com doenças hepáticas têm sido descritas por vários autores $^{(2,4,6,15,16,25,27,28,32,33,35)} \mathrm{em}$ todos os níveis do eixo hipotálamo-hipófise-tiróide, assim como no transporte e metabolismo periférico dos hormônios tiroidianos.

Há, nestes casos, uma multiplicidade de fatores envolvidos, dentre os quais, o tipo e o grau de desnutrição e comprometimento funcional hepático, com presença ou não de atividade inflamatória.

O presente trabalho estuda a função tiroidiana de um grupo de pacientes com hepatopatia crônica por vírus, com o

Serviços de Gastroenterologia do Hospital Universitário Clementino Fraga Filho do Departamento de Clínica Médica da Faculdade de Medicina da Universidade Federal do Rio de Janeiro - FM-UFRJ.

Serviço de Endocrinologia do Hospital Universitário Clementino Fraga Filho do Departamento de Clínica Médica da FM - UFRJ

Endereço para correspondência: Dr. Henrique Sergio Moraes Coelho - Rua Reseda, 23/301 - Lagoa - 22471-230 - Rio de Janeiro, RJ. 
objetivo de traçar perfil hormonal em indivíduos com diferentes graus de acometimento funcional hepático.

\section{PACIENTES E MÉTODOS}

No período entre 1993 e 1997, foram selecionados 125 pacientes com hepatopatia crônica causada pelos vírus da hepatite $\mathrm{B}$ ou $\mathrm{C}$, em acompanhamento no Serviço de Gastroenterologia do Hospital Universitário Clementino Fraga Filho (HUCFF) da Universidade Federal do Rio de Janeiro (UFRJ).

Todos os pacientes tinham diagnóstico histopatológico prévio de hepatite crônica ou cirrose hepática em material obtido através de biopsia hepática percutânea ou laparoscópica, segundo classificação vigente na época do diagnóstico ${ }^{(3)}$.

Os pacientes foram divididos em dois grupos distintos, de acordo com o diagnóstico histopatológico:

- pacientes com hepatite crônica ativa (HCA) - 31 casos;

- pacientes com cirrose pós-hepatite $(\mathrm{CPH})$ - 94 casos.

Os pacientes com cirrose hepática foram classificados do ponto de vista funcional, segundo Child-Pugh ${ }^{(5,10,31)}$, em A, B ou C, conforme e seguinte pontuação: A - 5 a 6 pontos, B - 7 a 9 pontos e C - 10 a 15 pontos.

Posteriormente, foram subdivididos em quatro grupos distintos: o grupo dos pacientes com HCA, que se manteve intacto, e o grupo dos pacientes com $\mathrm{CPH}$, divididos em três subgrupos diferentes:

- grupo I: 31 pacientes com $\mathrm{HCA}$ por vírus $\mathrm{B}$ ou $\mathrm{C}$;

- grupo II: 41 pacientes com $\mathrm{CPH}$ por vírus $\mathrm{B}$ ou $\mathrm{C}$, classificados em Child A

- grupo III: 35 pacientes com CPH por vírus B ou C, classificados em Child B;

- grupo IV: 18 pacientes com CPH por vírus $\mathrm{B}$ ou $\mathrm{C}$, classificados em Child C.

A etiologia da hepatopatia crônica foi avaliada segundo os seguintes critérios:

a) hepatopatia pelo vírus $\mathrm{B}$ - pacientes com $\mathrm{HBsAg}$ e $\mathrm{HBeAg}$ positivos pelo método ELISA (Abott);

b) hepatopatia pelo vírus $\mathrm{C}$ - Anti-HCV positivo por teste ELISA de segunda geração (Innotest-Innogenetics).

Foram excluidos deste estudo: pacientes com história de etilismo presente ou passado, aqueles com doenças sistêmicas que possam alterar os níveis dos hormônios tiroidianos, como diabetes mellitus, insuficiência renal, insuficiência cardíaca, os acometidos de patologias tiroidianas, pacientes que fazem uso de drogas capazes de alterar as dosagens hormonais ou os testes de função hepática, mulheres grávidas e pacientes com hepatite crônica ativa de qualquer outra etiologia.

Ao exame físico, deu-se particular importância à evidências de sinais de doença hepática ou tiroidiana, avaliação do grau de ascite e de encefalopatia hepática quando presentes.
Foram realizados os seguintes testes laboratoriais:

Testes de função hepática: albumina sérica - N: 3,5 - 4,8 g/dL (pelo método BCG); bilirrubina total - N: 0,2 - 1,0 mg/dL (pela técnica de Maccoy Evelyn modificada), tempo de atividade de protrombina (TAP) - N (em segundos): $<4,0$ seg. acima do controle (pelo método de Quick).

Testes de função tiroidiana (quimioluminescência): TSH - (Serono) - (N: 0,5-6,5 $\mu \mathrm{IU} / \mathrm{mL}) ; \mathrm{T} 4$ - (Serono) - (N: 4,5-12,5 $\mu \mathrm{g} / \mathrm{dL})$; T3 (DPC) - (N: 86-187 ng/dL); rT3 (T3 reverso) - (Serono) - (N: 9-35 ng/ dL); FT4 (T4 livre) - (DPC) - (N:0,8-2,0 ng/dL).

O teste do TRH consistiu na injeção intravenosa de $200 \mu \mathrm{g}$ de TRH com dosagem de TSH basal, 30, 60 e 90 min após. Foi considerada resposta normal quando o TSH elevou-se mais do que 2 $\mu \mathrm{U} / \mathrm{mL}$ e o pico foi maior do que $3,4 \mu \mathrm{U} / \mathrm{mL}$, ocorrendo aos 30 $\min ^{(17,30)}$.

Foi calculado o índice rT3/T3 (IrT3), sendo o valor normal considerado menor do que 0,5 .

De posse de todos estes dados, correlacionaram-se os achados histopatológicos e clínicos, com os resultados das dosagens bioquímicas e hormonais entre cada grupo de pacientes, divididos segundo a classificação de Child-Pugh ${ }^{(5,10,31)}$.

Foram utilizados os seguintes testes para comparação de médias e frequências : teste $t$ de Student, teste de Mann-Whitney, análise de variância "one way" ou análise não-paramétrica de Kruskal-Wallis, teste de comparações múltiplas de Bonferroni ou o teste de comparações múltiplas, baseado na estatística de Kruskal-Wallis, teste do qui-quadrado ou teste exato de Fisher, correlação de Pearson.

\section{RESULTADOS}

Dos 31 pacientes com HCA, 3 (9,7\%) foram classificados como hepatite crônica pelo vírus B e 28 (90,3\%) pelo vírus C. Dentre os 94 pacientes cirróticos, $26(27,7 \%)$ tiveram como etiologia de sua hepatopatia, o vírus B e 68 (72,3\%), o vírus C.

Dos 125 pacientes, $113(90,4 \%)$ apresentaram valores de TSH na faixa da normalidade, com apenas 11 pacientes $(8,8 \%)$ apresentando níveis elevados, sendo que destes, $6(54,5 \%)$ com diagnóstico de CPH-Child C.

O Gráfico 1, mostra que 43 pacientes $(34,4 \%)$ apresentaram T3 abaixo do normal, $9(7,2 \%)$ acima do normal e $73(58,4 \%)$ dentro da faixa normal. Considerando-se o valor de $136,5 \mathrm{ng} / \mathrm{dL}$ como o médio na variação de normalidade do exame, 88 pacientes (70,4\%) exibiram T3 normal-baixo ou abaixo do limite inferior da normalidade.

Dos 43 pacientes com T3 baixo, 2 (4,7\%) tinham o diagnóstico de HCA, 3 (7,0\%) de CPH-Child A e 20 (46,5\%) de CPH-Child B. Todos os 18 pacientes $(41,9 \%)$ com CPH-Child C tinham o T3 baixo. 


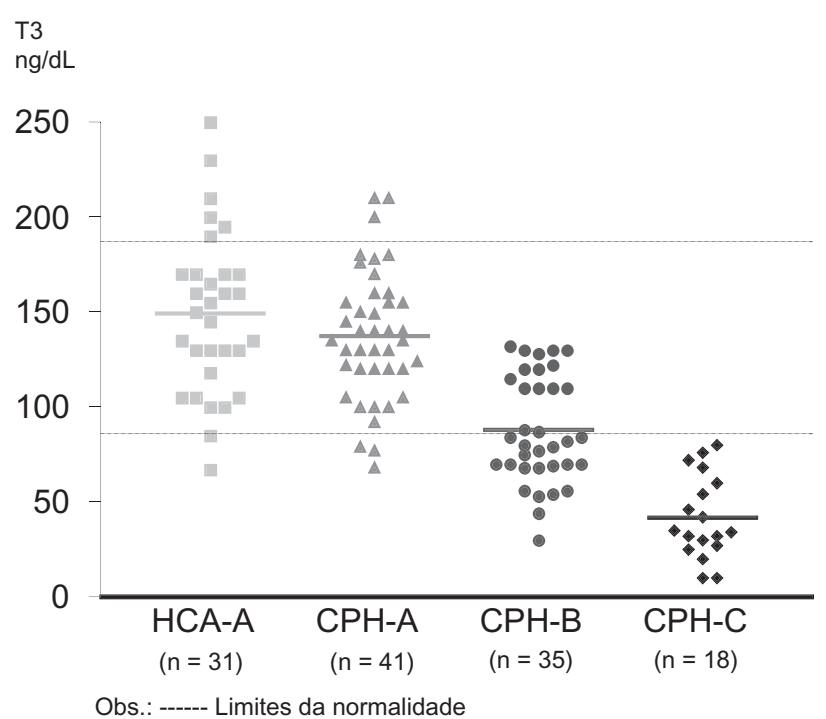

GRÁFICO 1 - Valores do T3 nos 125 pacientes estudados

Com relação ao T4, dentre os 125 pacientes estudados, $103(82,4 \%)$ apresentaram resultados dentro da faixa normal. Doze pacientes $(9,6 \%)$ exibiram níveis abaixo do normal, sendo que destes, $9(75 \%)$ pertencentes ao grupo com $\mathrm{CPH}-$ Child $\mathrm{C}$ e 10 pacientes (8\%), níveis acima do normal; destes, 7 (70\%) com diagnóstico de HCA ou CPH-Child A.

O T3 reverso (rT3) foi dosado em 122 pacientes, 83 (68\%) apresentando valores normais e $25(20,5 \%)$ resultados elevados em relação à população normal. Dentre estes últimos, 1 (4\%) tinha HCA, 7 (28\%) CPH-Child A, 7 (28\%) CPH-Child B e 10 (40\%) CPH-Child C. O Gráfico 2 mostra os resultados obtidos:

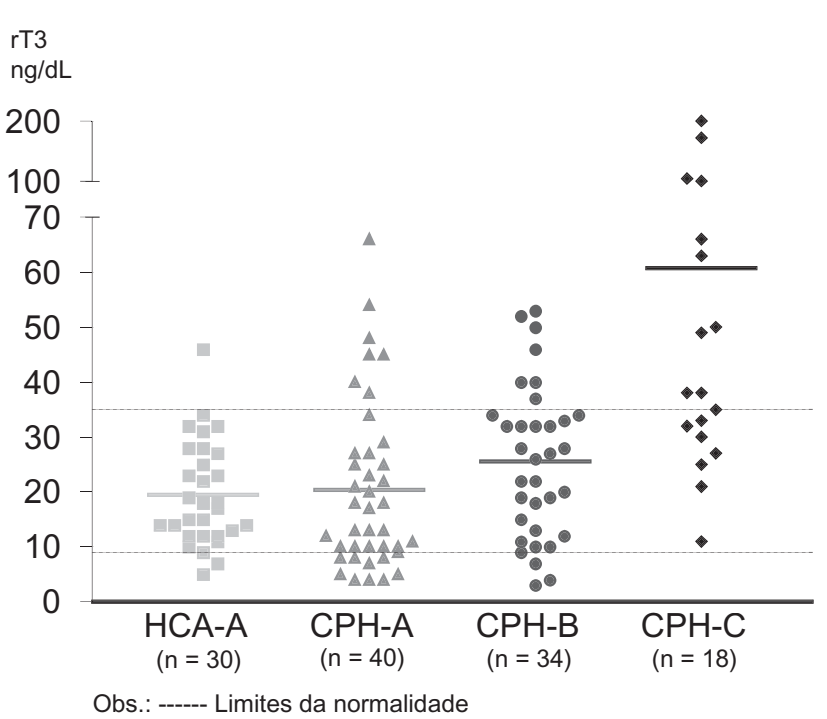

GRÁFICO 2 - Valores do rT3 nos 122 pacientes estudados
Dos 94 pacientes nos quais foram realizadas dosagens do T4 livre (FT4), em 78 pacientes $(81,9 \%)$ os valores foram normais. Doze pacientes $(12,8 \%)$, exibiram níveis inferiores à faixa da normalidade, sendo que destes, $8(66,6 \%)$ tinham CPH-Child C e em 5 pacientes (5,3\%), todos com HCA, os níveis foram superiores à faixa da normalidade.

O Gráfico 3 mostra correlação positiva significativa existente entre T3 e T4 séricos $(+0,68)(P=0,0001)$ e demonstra que os 3 pacientes $(2,4 \%)$, que exibiram T3 e T4 altos, tinham HCA ou CPHChild A. Em 12 pacientes (9,6\%), houve concomitância de T3 e T4 baixos. Destes, 3 (25\%) com diagnóstico de CPH-Child B e 9 (75\%) com CPH-Child C, estes últimos, evoluindo ao óbito em menos de 3 meses.

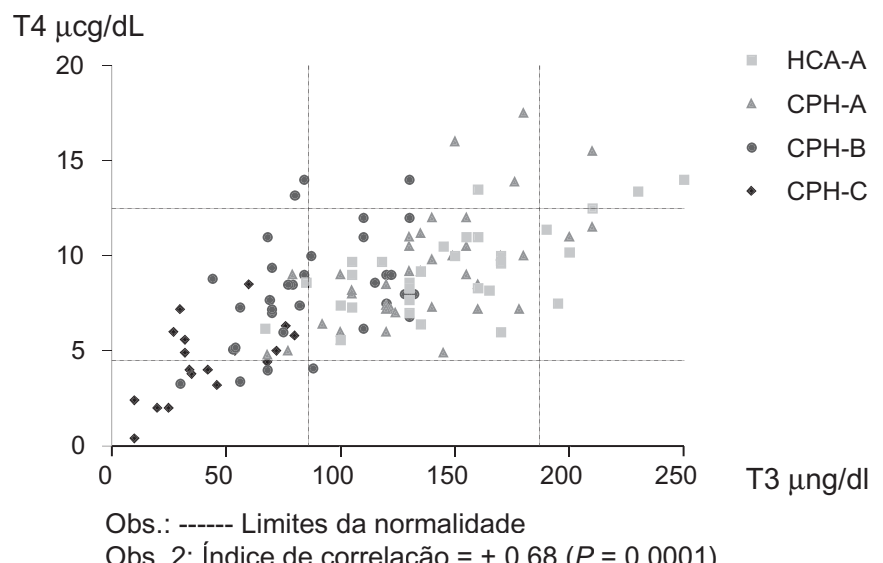

GRÁFICO 3 - Correlação entre valores de T3 e T4 nos 125 pacientes estudados

Houve também correlação positiva significativa existente entre T4 e FT4 $(+0,51)(P=0,0001)$. Observaram-se que 70 pacientes $(74,5 \%)$ exibiram valores normais de T4 e FT4; 5 (5,3\%) elevação do FT4 com valores normais do T4, todos com HCA; 11 (11,7\%) diminuição do FT4, sendo que destes, 7 (63,6\%) apresentaram também diminuição do T4 sérico, todos com CPH-Child C.

Foi observada também correlação negativa significativa existente entre T3 e rT3 séricos $(-0,35)(P=0,0001)$ e evidenciou-se presença de 16 pacientes $(13,1 \%)$ com elevação do rT3 simultânea a resultados baixos do T3, sendo que destes, 10 (62,5\%) com diagnóstico de $\mathrm{CPH}$ Child C.

A partir dos resultados do T3 e rT3 séricos, foi obtido índice rT3/ T3 (IrT3), calculado individualmente para cada paciente. O resultado normal para este IrT3 de acordo com HEPNER e CHOPRA ${ }^{(20)}$, seria inferior a 0,5 , com indicação de pior prognóstico quando superior a 5,0.

Nesta casuística, 3 pacientes $(2,5 \%)$ apresentaram valores superiores a 5,0, os 3 com diagnóstico de $\mathrm{CPH}$-Child $\mathrm{C}$, todos evoluindo ao óbito em menos de 3 meses da época da avaliação inicial. Dezessete 
pacientes $(13,9 \%)$ exibiram valores intermediários entre 0,5 e 5,0, todos com CPH-Child B ou C, 13 dos quais (76,5\%) evoluindo ao óbito em 1 ano. O restante dos pacientes, $102(83,6 \%)$, apresentaram valores normais do IrT3. (Gráfico 4).

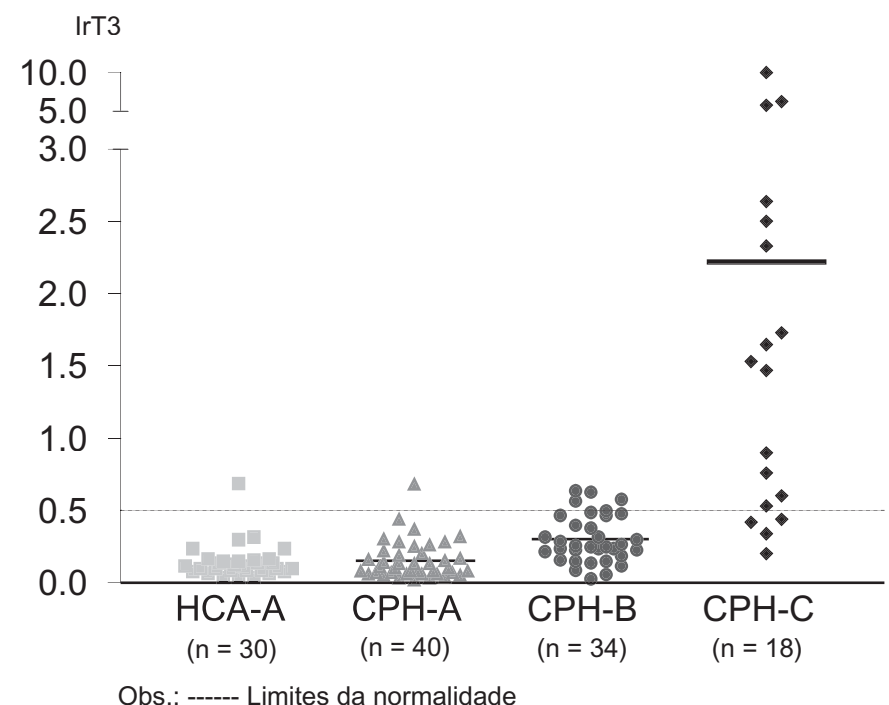

GRÁFICO 4 - Índice rT3/ T3 (IrT3)

O teste do TRH foi realizado em 86 pacientes. Destes, $76(88,4 \%)$ apresentaram resposta normal e $10(11,6 \%)$ além de TSH basal alto, resposta exagerada e prolongada à estimulação pelo TRH. Dentre estes últimos, 4 (40\%) tinham níveis normais de T3 e T4, todos com CPH-Child A, 1 (10\%) apresentava T3 baixo e T4 normal com diagnóstico de CPH-Child B e 5 (50\%) exibiram também níveis de T3 e T4 baixos, todos com CPH-Child C e evoluindo para óbito em 1 a 12 dias a partir da data de realização do exame.

As médias de cada dosagem hormonal foram calculadas inicialmente para o grupo de pacientes com HCA e CPH.

Houve diferença estatisticamente significativa entre as médias de todas as dosagens hormonais nos dois grupos. A média do TSH foi mais alta no grupo com $\mathrm{CPH}(P=0,02)$. As médias do T3, T4 e FT4, foram mais baixas no grupo com $\mathrm{CPH}(P=0,0001, P=0,02 \mathrm{e}$ $P=0,0003$, respectivamente), assim como as médias do rT3 e IrT3 foram mais elevadas neste grupo $(P=0,05$ e $P=0,001$, respectivamente).

Posteriormente, compararam-se as médias de acordo com as classes funcionais, conforme demonstra a Tabela 1.

De acordo com o exposto na Tabela 1, pode-se concluir que quando separamos os quatro grupos, apenas os pacientes do grupo $\mathrm{C}$ diferiram dos demais, com média do TSH mais alta $(P=0,004)$.

A média do FT4 dos pacientes com HCA foi expressivamente maior e a média dos pacientes com $\mathrm{CPH}-\mathrm{Child} \mathrm{C}$, menor, quando comparada aos demais $(P=0,0001)$.

Com relação à média do $\mathrm{T} 4$, apenas os pacientes do grupo $\mathrm{C}$ diferiram dos demais, com média do T4 mais baixa $(P=0,0001)$.

As médias do T3, rT3 e IrT3 foram significativamentes diferentes entre os grupos confrontados.

O T3 foi o exame que mais nitidamente se mostrou diferente entre os grupos. Mais baixo no grupo B do que no grupo A $(P=0,0001)$ e também acentuadamente mais baixo nos pacientes do grupo $\mathrm{C}$ quando comparados aos do grupo B $(P=0,0001)$.

Os pacientes do grupo $\mathrm{C}$ apresentaram média do rT3 expressivamente mais alta do que os demais grupos $(P=0,0001)$. Da mesma forma, o grupo $\mathrm{C}$, considerado individualmente, diferiu expressivamente dos demais, com média do $\operatorname{IrT} 3$ mais elevada $(P=0,0001)$.

\section{DISCUSSÃO}

A maioria dos pacientes que exibiram alterações nas dosagens séricas hormonais enquadrou-se na chamada "síndrome do T3 baixo", proposta pela classificação de CHOPRA et al. ${ }^{(9)}$, isto é, apresentaram T3 baixo, T4, TSH e teste do TRH normais, com impressão clínica de eutiroidismo.

Houve correlação entre os niveis de T3 sérico e o grau funcional hepático medido através a classificação de Child-Pugh. Os pacientes com HCA e CPH-A em sua imensa maioria ( $\pm 95 \%$ ) tinham valores de T3 normais, enquanto naqueles com Child B e $\mathrm{C}$ em mais de $40 \%$ dos casos o T3 sérico estava baixo. Isto foi particularmente evidente naqueles com CPH-C que apresentavam valores médios muito baixos $(41 \pm 21 \mathrm{ng} / \mathrm{mL})$.

O T3 foi o exame mais expressivo, estando baixo em 43 pacientes $(34,4 \%)$, isto sem considerarmos os 45 (36\%), cuja dosagem esteve próxima do limite inferior da normalidade, totalizando 88 pacientes

TABELA 1 - Comparação das médias hormonais entre as quatro classes funcionais

\begin{tabular}{lcccccc}
\hline & TSH & T3 & T4 & FT4 & rT3 & IrT3 \\
HCA & $1,9 \pm 1,4$ & $149,2 \pm 42,3$ & $9,3 \pm 2,2$ & $1,7 \pm 0,6$ & $19,5 \pm 9,5$ & $0,2 \pm 0,1$ \\
CPH-Child A & $3,0 \pm 3,8$ & $137,4 \pm 34,2$ & $9,3 \pm 2,9$ & $1,2 \pm 1,3$ & $20,4 \pm 15,4$ & $0,2 \pm 0,1$ \\
CPH-Child B & $2,7 \pm 1,9$ & $88,0 \pm 28,4$ & $8,3 \pm 2,8$ & $1,3 \pm 0,4$ & $25,6 \pm 13,9$ & $0,3 \pm 0,2$ \\
CPH-Child C & $7,2 \pm 11,5$ & $41,8 \pm 21,9$ & $4,5 \pm 2,0$ & $0,7 \pm 0,4$ & $60,8 \pm 52,1$ & $2,2 \pm 2,6$ \\
$P=0,004$ & $P=0,0001$ & $P=0,0001$ & $P=0,0001$ & $P=0,0001$ & $P=0,0001$ \\
\hline
\end{tabular}


(70,4\%) com T3 normal-baixo ou abaixo do limite inferior da normalidade. Estes achados corroboram a hipótese de que níveis séricos baixos de T3 parecem se correlacionar bem com a gravidade da doença hepática $^{(13,21,22)}$.

A correlação positiva significativa $(+0,68)(P=0,0001)$ existente entre os valores de T3 e T4 evidenciou concomitância de níveis séricos baixos de ambos, em 12 casos (9,6\%). Destes, três (25\%) com CPH$\mathrm{B}$ e nove (75\%) com CPH-C, estes últimos, evoluindo para óbito em menos de 3 meses. Estes pacientes, de acordo com CHOPRA et al. ${ }^{(9)}$, enquadram-se na chamada "síndrome do T3 e T4 baixos". São geralmente muito graves e os níveis séricos de T4 parecem ter correlação com o prognóstico e mortalidade da doença.

A maior parte dos pacientes desta série $(90,4 \%)$ apresentou valores absolutos do TSH dentro da faixa da normalidade, sendo que apenas os pacientes com CPH-C tiveram médias de TSH elevada $(7,2 \pm 11,5$ $\mathrm{mUI} / \mathrm{mL}$ ). Geralmente, o TSH sérico é normal em patologias não tiroidianas $^{(19,26,27,40)}$. Deve-se ter em mente, no entanto, que o controle da secreção do TSH nesses pacientes pode ser influenciado por forças opostas $^{(1)}$ (febre e estresse como inibidores e hipotermia, T3 e T4 baixos como estimuladores) e, assim, o nível de TSH "normal" resultante pode ser mau interpretado e este paciente pode estar, transitoriamente, em "hipotiroidismo" fisiológico ${ }^{(41)}$. Em contraste com a maioria das outras patologias que cursam com a "síndrome do T3 baixo", pacientes com CPH e T3 sérico baixo podem exibir freqüentemente TSH basal elevado. Este nível não se correlaciona necessariamente com os níveis baixos do $\mathrm{T} 3^{(6,27)}$, mas parece se correlacionar com a gravidade da doença hepática ${ }^{(40)}$, fato este observado neste estudo, já que a média do TSH no grupo com $\mathrm{CPH}$ $\mathrm{C}$ foi significativamente mais alta do que nos demais grupos $(P=$ $0,004)$

O rT3 esteve elevado em 25 pacientes (20,8\%). Destes, 10 (40\%) com $\mathrm{CPH}-\mathrm{C}$, que diferiram significativamente dos demais, com elevação desta média (CPH-C: 60,8 $\pm 52,1 \mathrm{ng} / \mathrm{dL}$; CPH-B: 25,6 \pm 13,9 ng/dL; CPH-A: 20,4 $\pm 15,4$ ng/dL; HCA: 19,5 \pm 9,5 ng/dL) $(P=0,0001)$. Estes resultados estão de acordo com a literatura ${ }^{(4,15}$ $16,27,41)$, pois a deiodenase é responsável tanto pela metabolização periférica do T4 em T3, quanto pela degradação do rT3. Nas doenças sistêmicas, há diminuição da síntese de T3 e acúmulo do rT3 na circulação. Como esta enzima está preferencialmente localizada no fígado e nos rins ${ }^{(20)}$, as alterações metabólicas tornam-se mais evidentes nas hepatopatias. A dosagem do nível sérico do rT3 não só se correlaciona bem com a gravidade da doença hepática ${ }^{(13,21,22)}$, mas também tem sido utilizada clinicamente como indicador prognóstico da sobrevida de pacientes com doença hepática crônica, identificando os de maior risco de evoluir a óbito antes ou imediatamente após o transplante hepático ${ }^{(20,39)}$

O IrT3 (IrT3/T3) foi calculado no intuito de acrescentar outro critério indicativo do prognóstico para estes pacientes ${ }^{(20)}$. Nota-se na presente casuística, que dos 20 pacientes $(16,4 \%)$ que exibiram elevação deste índice, 3 (15\%) apresentavam valores superiores a 5,0, indicando péssimo prognóstico, todos com $\mathrm{CPH}-\mathrm{C}$ e evoluindo para óbito em menos de 3 meses. Já 17 (85\%) exibiram valores intermediários entre 0,5 e 5,0, indicativos de mau prognóstico, todos com diagnóstico de CPH-B (23,5\%) ou CPH-C (64,7\%), 13 dos quais (76,5\%) evoluindo para óbito no período de 1 ano. Este índice calculado foi utilizado por HEPNER e CHOPRA ${ }^{(20)}$, em 1979, para avaliação da gravidade da hepatopatia alcoólica e, mais recentemente, tem mostrado valor preditivo em pacientes aguardando o transplante hepático ${ }^{(39)}$.

Os pacientes com $\mathrm{CPH}$, apresentaram em média, valores reduzidos de FT4.

Estes resultados são compatíveis com a hipótese de CHOPRA et al. ${ }^{(7,8)}$, posteriormente confirmada por outros investigadores como KAPTEIN et al. ${ }^{(23)}$ e WOEBER e MADDUX ${ }^{(42)}$, da presença de um fator de inibição da ligação T4-TBG nas doenças crônicas, com conseqüente aumento dos níveis circulantes do hormônio livre. Nos pacientes com CPH-C, foi comum a observação de níveis séricos reduzidos de FT4 tanto em valores absolutos (44,4\%), quanto em média $(0,7 \pm 0,4 \mathrm{ng} / \mathrm{dL})(P=0,0001)$. Não foi encontrado na literatura qualquer nota que reforce este achado, correlacionando níveis baixos do FT4 com gravidade da hepatopatia crônica, mas ele está de acordo com a verificação do $\mathrm{T} 4$ sérico também muito baixo neste grupo de pacientes mais graves ${ }^{(13,24,36)}$.

A correlação positiva significativa $(+0,51)(P=0,0001)$ existente entre os valores de T4 e FT4 vem corroborar esta hipótese, pois os 5 pacientes $(5,3 \%)$ que exibiram elevação do FT4 com valores normais de T4 tinham HCA, porém, dos 12 pacientes (12,6\%) que apresentaram redução do FT4, 7 (58,3\%) tinham também diminuição do T4 sérico, todos com $\mathrm{CPH}-\mathrm{C}$.

O teste do TRH foi normal na maior parte dos pacientes desta série $(88,4 \%)$, achado este compatível com vários estudos publicados ${ }^{(6,}$ 19, 38, 41). Em 10 pacientes (11,6\%), além de um TSH basal elevado, observou-se resposta exagerada e prolongada à estimulação pelo TRH. Apesar de vários relatos existentes na literatura de uma resposta ao teste do TRH exagerada e prolongada em pacientes com hepatopatia crônica $^{(9,16,17,30,33,34)}$ e da proposição feita por GREEN et al. ${ }^{(16)}$ de que seja provável que, em alguns pacientes com cirrose, exista um distúrbio funcional do hipotálamo, levando a um defeito na regulação da secreção do TSH, não há relatos de que isto possa estar relacionado com a gravidade da doença. Isto foi observado neste estudo, principalmente nos pacientes com CPH-C, que exibiram elevação do TSH basal e resposta exagerada e prolongada do teste do TRH, com T3 e T4 baixos, todos evoluindo para óbito em média em 6 dias após a data do exame. No entanto, não se pode descartar que estes pacientes possam ter comprometimento da função tiroidiana por doença primária da glândula. 
Além do teste do TRH normal na grande maioria dos pacientes estudados, as dosagens normais de T4 e FT4 corroboram a hipótese de eutiroidismo. Por outro lado, o hipotiroidismo também foi afastado, pelos resultados normais do TSH basal e normais-altos do rT3 sérico. Vários autores, dentre os quais CHOPRA et al. ${ }^{(9)}$ e OPPENHEIMER ${ }^{(29)}$, sugerem a suspeita de hipotiroidismo na vigência de doenças crônicas, apenas quando o TSH basal for superior a $15 \mu \mathrm{IU} / \mathrm{mL}$. O T3 não tem valor para o diagnóstico de hipotiroidismo nas doenças não-tiroidianas pois, como já foi visto, é freqüente a "síndrome do T3 baixo". A concentração sérica do rT3 é diretamente proporcional à secreção tiroidiana de T4, com o aumento de sua secreção no hipertiroidismo e redução no hipotiroidismo. Os resultados deste estudo, com 108 pacientes $(88,5 \%)$ exibindo rT3 normal ou elevado, praticamente afastam a hipótese de hipotiroidismo nesses pacientes.

Assim, conclui-se que: 1) são frequentes as alterações no metabolismo dos hormônios tiroidianos na hepatopatia cônica por vírus; 2) há relação direta entre os níveis séricos baixos do T3 e elevados do rT3 e IrT3 com o grau de disfunção hepática, segundo a classificação de Child-Pugh; 3) a "síndrome do T3 baixo" foi a manifestação prevalente dentre as alterações hormonais neste grupo de pacientes, sendo o T3 sérico o exame mais expressivo, gradativamente mais baixo quanto mais avançada a disfunção hepatocelular, diferenciando as classes funcionais entre si. Além do teste do TRH normal na maioria dos pacientes, as dosagens normais do T4 e FT4 corroboram a hipótese de eutiroidismo; 4) resposta exagerada e prolongada ao teste do TRH foi observada principalmente em alguns pacientes com CPH-C, todos apresentando elevação do TSH basal, além de T3 e T4 baixos. Estes achados podem estar relacionados com a gravidade da hepatopatia, embora não se possa descartar doença primária tiroidiana; 5) a concomitância de níveis séricos mais altos de $\mathrm{T} 3$ e T4 nos pacientes com CPH-C, é indicativo de mau prognóstico a curto prazo.

Novis M, Vaisman M, Coelho HSM. Thyroid function tests in patients with viral chronic liver disease. Arq Gastroenterol 2001;38(4):254-260.

ABSTRACT - Background - One hundred and twenty five patients with virus B or C chronic active hepatitis and postnecrotic cirrhosis and different degrees of liver dysfunction were studied. Aim - 1) To determine a thyroid hormonal profile; 2) to evaluate the prognostic value of these tests in relation to the progression of the disease and mortality; 3) compare these findings with Child-Pugh classification. Patients and Methods - The patients were divided in four groups: a) 31 with chronic active hepatitis; b) 41 with postnecrotic cirrhosis Child A; c) 35 with postnecrotic cirrhosis Child B and d) 18 with postnecrotic cirrhosis Child C. The protocol comprised serum measurements of albumin and bilirrubin, estimates of prothrombin time and clinical evaluation of ascites and encephalopathy, measurement of total serum triiodothyronine, thyroxine, thyroid-stimulating hormone, free thyroxine, reverse triiosothyronine, calculated rT3/T3 index (IrT3) and thyrotropin-releasing hormone test. Results - Total serum triiodothyromnine showed the most significant difference among the groups, gradually lower as the disease became more advanced (CAH: 149,2 $\pm 42,3 \mathrm{ng} / \mathrm{dL}$; PNC-A: 137,4 $\pm 37,2 \mathrm{ng} / \mathrm{dL} ; \mathrm{PNC}-\mathrm{B}: 88,0 \pm 28,4 \mathrm{ng} / \mathrm{dL}$ and PNC-C: 41,8 \pm $21,9 \mathrm{ng} / \mathrm{dL})$. Low levels of T4 (4,5 $\pm 2,0 \mu \mathrm{g} / \mathrm{dL})$ and FT4 $(0,7 \pm 0,4 \mathrm{ng} / \mathrm{dL})$ and elevated levels of thyroid-stimulating hormone (7,2 $\pm 11,5$ $\mu I U / m L)$, reverse triiosothyronine $(60,8 \pm 52,1 \mathrm{ng} / \mathrm{dL})$ and calculated $r T 3 / T 3$ index $(2,2 \pm 2,6)$ were more frequent in patients with postnecrotic cirrhosis Child C. Thyrotropin-releasing hormone test was normal in the majority of the patients. Conclusion - The present study shows a positive relationship between the low serum levels of T3 and elevated serum levels of rT3 and IrT3/T3 with the degree of hepatic dysfunction according to the Child-Pugh classification.

HEADINGS - Thyroid function tests. Hepatitis, chronic. Liver cirrhosis.

\section{REFERÊNCIAS BIBLIOGRÁFICAS}

1. Bacci V, Shussler GC, Kaplan TB. The relationship between serum triiodothyronine and thyrotropin during systemic illness. J Clin Endocrinol 1982;54:1229.

2. Bianchi GP, Zoli M, Marchesini G, Volta U, Vecchi F, Iervese T, Bonazzi C, Pisi E. Thyroid gland size and function in patients with cirrhosis of the liver. Liver 1991;11:71.

3. Bianchi L, De Groote J, Desmet VJ. Acute and chronic hepatitis revisited. Review by an international group. Lancet 1977;2:914.

4. Borzio M, Caldara R, Borzio F, Piepoli V, Rampini P, Ferrari C. Thyroid function tests in chronic liver disease: evidence for multiple abnormalities despite clinical euthyroidism. Gut 1983;24:631.

5. Child CG III, Turcotte J. The liver and portal hypertension. Philadelphia: WB Saunders; 1964. p.50
6. Chopra IJ, Solomon DH, Chopra U, Young RT, Chua Teco GN. Alterations in circulating thyroid hormones and thyrotropin in hepatic cirrhosis: evidence for euthyroidism despite subnormal serum triiodothyronine. J Clin Endocrinol Metab 1974;39:501.

7. Chopra IJ, Teco GN, Nguyen AH, Solomon DH. In search of an inhibitor of thyroid hormone binding to serum proteins in nonthyroid illnesses. J Clin Endocrinol Metab 1979;49:63.

8. Chopra IJ, Solomon DH, Hepner GW, Morgenstein AA. An inhibitor of the binding of thyroid hormones to serum proteins is present in extra thyroidal tissues. Science 1982;215:407.

9. Chopra IJ, Hershman JM, Pardridge WM, Nicoloff JT. Thyroid function in nonthyroidal illnesses (UCLA Conference). Ann Intern Med 1983;96:946.

10. Christensen E, Schilichting P, Fauerholdt L, Gluud C, Andersen PK, Juhl E, Poulsen H, Tygstrup N. Prognostic value of Child-Turcotte criteria in medically treated cirrhosis. Hepatology 1984;4:430. 
11. Custro N, Scafidi V, Notarbartolo A. Alterations in circadian rhythm of serum thyrotropin in critically ill patients. Acta Endocrinol 1992;127:18.

12. Custro N, Scafidi V, Gallo S, Notarbartolo A. Deficient pulsatile thyrotropin secretion in the low-thyroid-hormone state of severe nonthyroidal illness. Eur J Endocrinol 1994;130:132.

13. D'azzo G, Pinzelo GB, Pace F, Garofalo P, Craxi A, Janni A. Prognostic value of thyroid function tests in predominantly nonalcoholic cirrhotic patients: a prospective investigation. J Endocrinol Invest 1985;8:331

14. Engstrom WW, Markardt B. The efects of serious illness and stress on the circulating hormone. J Clin Endocrinol Metab 1955;15:953.

15. Faber J, Thomsen HF, Lumholtz IB, Kirkegaard C, Siersbaek Nielsen K, Friis T. Kinetic studies of thyroxine, 3,5,3'-triiodothyronine, 3,3',5'-triiodothyronine, 3'5'-diiodothyronine, 3,3'-diiodothyronine and 3'-monoiodothyronine in patients with liver cirrhosis. J Clin Endocrinol Metab 1981;53:978.

16. Green JRB, Snitcher EJ, Mowat NA, Ekins RP, Rees LH, Dawson AM. Thyroid function and thyroid regulation in euthyroid men with chronic liver disease: evidence of multiple abnormalities. Clin Endocrinol (Oxf) 1977;7:453.

17. Hall R, Ormston BJ, Besser GM, Cryer RJ. The thyrotrophin-releasing-hormone test in diseases of the pituitary and hypothalamus. Lancet 1972;1:759.

18. Hashimoto T, Kawai K, Nishibu M, Fujita S, Horita H. Clinical evaluation of accuraci in determining serum free thyroxine and free triiodothyronine in patients with nonthyroidal illness: immunoglobulin effect on T3/TBG ratio and T4/TBG ratio. Endocrinol Japon 1991;38:633.

19. Hasselbalch HC, Bech K, Eskildsen PC. Serum prolactin and thyrotropin responses to thyrotropin-releasing-hormone in men with alcoholic cirrhosis. Acta Med Scand 1981;209:37.

20. Hepner GW, Chopra IJ. Serum thyroid hormone levels in patients with liver disease. Arch Intern Med 1979;139:1117.

21. Israel Y, Walfish PG, Orrego H, Blake J, Kalant H. Thyroid hormones in alcoholic liver disease: effect of treatment with 6-N-propylthiouracil. Gastroenterology 1979;76:116.

22. Kabadi UM, Prema Chandra BN. Serum T3 and reverse T3 levels in hepatic cirrhosis: relation to hepatocellular damage and normalization on improvement of liver disease. Am J Gastroenterol 1983;78:750.

23. Kaptein EM, Robinson WJ, Grieb DA, Nicoloff JT. Peripheral serum thyroxine, triiodothyronine and reverse triiodothyronine kinetics in the low thyroxine state of acute nonthyroidal illnesses. J Clin Invest 1982;69:526.

24. Kodding R, Hesch RD. The importance of iodothyronine in severe nonthyroida disease in the low T3 syndrome. In: Hesch RD, editor. Portal hypertention. London: Academic Press; 1981. p.187.

25. L'Age M, Meinhold H, Wenzel KW, Schleusener H. Relations between serum levels of TSH, TBG, T4, T3, rT3 and various histologically classified chronic liver diseases. J Endocrinol Invest 1980;3:379.

26. Nomura S, Chambers JB Jr, Buck MW, Pittman CS, Shimizu T. The peripheral convertion of thyroxine (T4) to triiodothyronine (T3 ) in liver patients. Clin Res 1974;22:62 A.
27. Nomura S, Pittman CS, Chambers JB, Buck MW, Shimizu T. Reduced peripheral convertion of thyroxine to triiodothyronine in patients with hepatic cirrhosis. J Clin Invest 1975;56:643.

28. Oppenheimer JH. An unsolved problem: low serum PBI values in patients with chronic disease. J Chron Dis 1969;22:129.

29. Oppenheimer JH. Thyroid function tests in nonthyroidal disease. J Chron Dis 1982;35:697.

30. Ormston BJ, Cryer RJ, Garry R, Besser GM, Hall R. Thyrotropin-releasinghormone as a thyroid function test. Lancet 1971;2:10.

31. Pugh RNH, Murray-Lyon IM, Dawson SL, Pietroni ML, Williams R. Transection of the oesophagus for bleeding oesofageal varices. Br J Surg 1973;60:646.

32. Rink C, Siersleben U, Hearting J, Mende T, Nilius R. Development of the low T3 syndrome and prognosis assesment in patients with liver cirrhosis. Gastroenterology 1991;51:138.

33. Schussler GC, Schaffner F, Korn F. Increased serum thyroid hormone binding and decreased free hormone in chronic active liver disease. N Engl J Med 1978;299:510.

34. Sheridan P. Thyroid hormones and the liver. Clin Gastroenterol 1983;12:797.

35. Sifré RB, Silvestre FM, Garcia AG, Elkak R, Garcia MDA. Interacciones tiróideshígado en medicina clínica. Rev Enferm Ap Dig 1988;73:315.

36. Slag MF, Morley JE, Elson MK, Crowson TW, Nuttall FQ, Shafer RB. Hypothyroxinemia in critically ill patients as a predictor of high mortality. JAMA 1981;245:43.

37. Suzuki Y, Nanno M, Gemma R, Yoshimi T. Plasma free fatty acids, inhibitor of extrathyroidal convertion of T4 to T3 and thyroid hormone binding inhibitor in patients with various nonthyroidal illnesses. Endocrinol Japon 1992;139:445.

38. Van Thiel DH, Smith WI Jr, Wrigth C, Abuid J. Elevated basal and abnormal thyrotropin releasing hormone - induced thyroid stimulating hormone secretion in chronic alcoholic men with liver disease. Alcohol Clin Exp Res $3: 302,1979$

39. Van Thiel DH, Udani M, Schade RR, Sanghui A, Starzl TE. Prognostic value of thyroid hormone levels in patients evaluated for liver transplantation. Hepatology 1985;5:862.

40. Van Thiel DH. Disorders of the hypothalamic-pituitary-gonadal and thyroida axis in patients with liver disease. In: Zakim D, Boyer, editors. Hepatology. A textbook of liver disease. 2 ed. Philadelphia: WB Saunders; 1989. p. 513.

41. Wartofsky L, Burman KD. Alterations in thyroid function in patients with systemic illness: the "euthyroid sick syndrome". Endocr Rev 1982;3:164.

42. Woeber KA, Maddux BA. Thyroid hormone binding in nonthyroid illness. Metabolism 1981;30:412. 